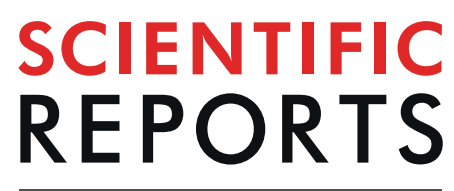

natureresearch

\title{
Low skeletal muscle area as a prognostic marker for chronic obstructive pulmonary disease in elderly patients admitted to ICU
}

\begin{abstract}
Jiehua Zhi ${ }^{1,4}$, Qing Shan ${ }^{2,4}$, Lanyu Liang ${ }^{2 *}$, Han Liv² \& Hua Huang ${ }^{3}$
Low L3 skeletal muscle area (SMA), which is assessed on computed tomography (CT) images, has been reported to indicate poor clinical outcomes of patients with acute exacerbation of chronic obstructive pulmonary disease (COPD). The dorsal muscle group area at the T12 vertebral level (T12DMA) was used as an alternative to L3 SMA. This study aimed to investigate whether T12DMA could be used as a predictor of in-hospital mortality and long-term survival in elderly patients with COPD admitted to the intensive care unit (ICU). This single-center retrospective case-control study was performed by analyzing the clinical information and measuring T12DMA on chest CT images of elderly patients with COPD admitted to the ICU between May 2013 and May 2018. This study included 136 patients. The multivariate logistic regression analysis showed that T12DMA, neutrophil-lymphocyte ratio, invasive mechanical ventilation, and systemic steroid therapy were independent risk factors for predicting the hospital mortality. The median survival was significantly higher in the high-T12DMA group (214 days) than in the low-T12DMA group (32 days).
\end{abstract}

Chronic obstructive pulmonary disease (COPD) represents a major public health problem worldwide due to increasing prevalence, morbidity, and mortality. It was ranked the eighth among the causes of disease burden worldwide measured by disability-adjusted life-years in $2015^{1}$. It was also the fourth leading cause of years of life lost in China in $2017^{2}$. The prevalence of COPD was $14 \%$ in patients aged $\geq 65$ years, with a fold increase for every 10 -year increment of age ${ }^{3}$. COPD often presents in aging patients as a component of multimorbidity. Sarcopenia was one of the common comorbidities ${ }^{4}$. Sarcopenia, which is defined as age-associated loss of muscle mass and strength, is highly prevalent among patients with COPD and is associated with a worse prognostic index ${ }^{4-6}$. The prevalence of sarcopenia was reported to be $24 \%$ among patients with COPD based on a cross-sectional study of a Southeast Asian population. It was associated with age and Global Initiative for Chronic Obstructive Pulmonary Disease (GOLD) stage ${ }^{7}$. Patients with COPD worsened by respiratory failure requiring mechanical ventilation (MV) were more likely to be admitted to the intensive care unit (ICU). Sarcopenia was a strong predictor of mortality among patients with COPD or other diseases requiring MV in the $\mathrm{ICU}^{8-11}$. Meanwhile, muscle wasting, as a major contributor to ICU-acquired weakness, occurred early and rapidly during the first week of critical illness $^{12}$. It was associated with short-term and long-term mortality, as a driver of long-term functional disability after discharge from the ICU ${ }^{13-15}$. Therefore, the diagnosis of sarcopenia might help in preventing the mortality of these patients.

In 2018, the European Working Group on Sarcopenia in Older People (EWGSOP) revised the diagnostic criteria for sarcopenia, which is now determined by both low muscle quantity and quality. The EWGSOP recommends the area measurement on a single cross-section computed tomography (CT) image at the level of the third lumbar (L3) vertebra as an alternative tool, since this area accurately represents the whole-body muscle. Thus, this method may serve as an alternative tool for assessing muscle quantity ${ }^{16}$. The quantification of L3 skeletal muscle area (SMA) using a single transverse CT slice showed that more than $56 \%$ of patients with respiratory failure had comorbidity of sarcopenia ${ }^{8}$. However, abdominal CT is not a routine examination for patients with COPD.

${ }^{1}$ Department of Gastroenterology, The Affiliated Hospital of Yangzhou University, Yangzhou University, Yangzhou, China. ${ }^{2}$ Department of Gerontology, The Affiliated Hospital of Yangzhou University, Yangzhou University, Yangzhou, China. ${ }^{3}$ Department of Radiology, The Affiliated Hospital of Yangzhou University, Yangzhou University, Yangzhou, China. ${ }^{4}$ These authors contributed equally: Jiehua Zhi and Qing Shan. *email: Ilywind1026@163.com 
Chest CT scanning is a conventional assessment of muscle mass and is easy to acquire for patients with COPD. The examination of T12, T11, and T10 may serve as an alternative strategy when L3 is unavailable ${ }^{17}$. Moreover, the decline in paravertebral muscle size and attenuation at T12 on CT images has been reported to be associated with mortality among patients with hip fracture ${ }^{18}$ and those undergoing liver transplantation ${ }^{19}$ and general and vascular surgery ${ }^{20}$. The relationship between the T12 vertebral level (T12DMA) and the mortality related to the acute exacerbation of COPD (AECOPD) remains unclear.

This study retrospectively investigated clinical parameters, including T12DMA, dorsal muscle group (DMG) density at the T12 vertebral level (T12DMD), and other risk factors, to evaluate whether these parameters might serve as prognostic markers for in-hospital mortality among elderly patients with AECOPD requiring ICU admissions for ventilation support. It also explored whether the level of T12DMA at admission might be a predictor of long-term survival among patients with COPD after ICU admission.

\section{Materials and Methods}

Population cohort and design. This was a retrospective case-control study conducted by the Affiliated Hospital of Yangzhou University, a Chinese tertiary teaching hospital, between February 2013 and May 2018. Patients diagnosed with AECOPD based on the International Classification of Diseases, Tenth Revision codes, including J44.000, J44.101, and J44.100, were consecutively enrolled. All patients were previously diagnosed with COPD based on the GOLD 2017 recommendations. Patients had dyspnea with or without unconsciousness when admitted to the ICU directly from the emergency department or during hospitalization.

The inclusion criteria were as follows: (1) age $\geq 60$ years; (2) invasive mechanical ventilation (IMV) or noninvasive mechanical ventilation (NIMV) during the ICU stay; and (3) chest CT scan performed at admission or within $48 \mathrm{~h}$ after admission. The exclusion criteria were as follows: (1) discharge within $24 \mathrm{~h}$; (2) no adequate imaging within $48 \mathrm{~h}$ after admission; (3) patients admitted to the ICU due to other diseases and suffering from comorbidity of COPD; (4) tumor history; (5) an ICU admission history; and (6) more than 90 days of ICU stay.

A total of 136 patients were eligible for this study. They were divided into two groups based on their survival when discharged from the hospital. All enrolled patients were followed up until October 31, 2019.

Demographic and clinical information. Data on demographic statistics, comorbidities, clinical information, laboratory parameters, and in-hospital mortality were abstracted from the electronic medical records. The death date of patients was acquired from the National Population Information Database.

The exposure variables were collected, including sex, age, smoking status, Charlson Comorbidity Index $(\mathrm{CCI})^{21}$, APACHE II scores, Glasgow Coma Score (GCS), NIMV or IMV, administration route of steroids [systemic corticosteroid (SCS) treatment and non-SCS treatment (treatment with inhaled or oral corticosteroids], hospital mortality. White blood cell (WBC) count, neutrophil count, lymphocyte count, platelet count, $\mathrm{pH}$, $\mathrm{PCO}_{2}$, and $\mathrm{PaO}_{2} / \mathrm{FiO}_{2}$, were recorded within $24 \mathrm{~h}$ of admission to the ICU. Neutrophil-lymphocyte ratio (NLR) and platelet-lymphocyte ratio (PLR) were calculated within $24 \mathrm{~h}$ of admission to the ICU. The serum albumin recorded at admission $\left(\right.$ Albumin $\left._{\mathrm{ad}}\right)$, the minimum value during hospitalization $\left(\right.$ Albumin $\left._{\min }\right)$, and the difference between Albuminad and Albuminmin (termed as $\Delta$ Albumin) were measured.

Management of MV during the ICU stay. The selection of mechanical ventilation procedures was initiated based on a decision made by the staff physician according to the symptoms and pathophysiological components. Hypercapnic respiratory failure with a $\mathrm{pH}<7.35$ was the major criterion based on which the physician initiated the NIMV procedure. The major criteria of IMV were as follows ${ }^{22}$ : (1) severe dyspnea involving accessory muscles and paradoxical abdominal motion; (2) respiratory arrest; (3) loss of consciousness; (4) failure of noninvasive treatment; (5) psychomotor agitation requiring sedation; and (6) hemodynamic instability with a systolic blood pressure less than $70 \mathrm{~mm} \mathrm{Hg}$ or greater than $180 \mathrm{~mm} \mathrm{Hg}$.

Diagnosis of pneumonia. Pneumonia was diagnosed based on the chest CT scan at admission. The CT scan was reviewed by an experienced radiologist. Pulmonary edema, pulmonary fibrosis, tuberculosis, lung cancer, and pulmonary embolism were excluded.

Measurement of T12DMA and T12DMD. The electronically stored CT images of patients at admission or within $48 \mathrm{~h}$ after admission were analyzed using Picture Archiving and Communication Systems (PACS, IMPAX6.3.1.4095, AGFA HealthCare NV, Belgium.). T12DMA was defined as any muscle within the region posterior to the T12 spine and ribs and no more lateral than the lateral-most edges of the erector spinal muscles ${ }^{19}$ (Fig. 1). All imaging analyses were conducted in the cross-sectional area at the level of the 12th thoracic spine and manual sketch ridge of the DMG. The muscle area and density were evaluated using the PACS. T12DMA was recorded as the sum of bilateral DMG area, while T12DMD was recorded as the mean of bilateral DMG radiodensity. The measurements of T12DMA and T12DMD were independently performed by an experienced radiologist and a well-trained doctor, and the final measurements were the average of the two.

Ethical approval. This study was carried out in accordance with the declaration of Helsinki (2000) of the World Medical Association. This was a retrospective trial. The Human Research Ethics Committee of the Affiliated Hospital of Yangzhou University that approved this study also waived the need for informed consent.

Statistical analysis. Statistical analyses were performed using SPSS 23.0 (IBM, NY, USA). All demographic and clinical characteristics were compared between the two groups based on the status at discharge (surviving or deceased).

All variables were evaluated for normal distribution using the Shapiro-Wilk test. All quantitative variables in line with the normal distribution were described as mean \pm standard deviation. The univariate analysis was 


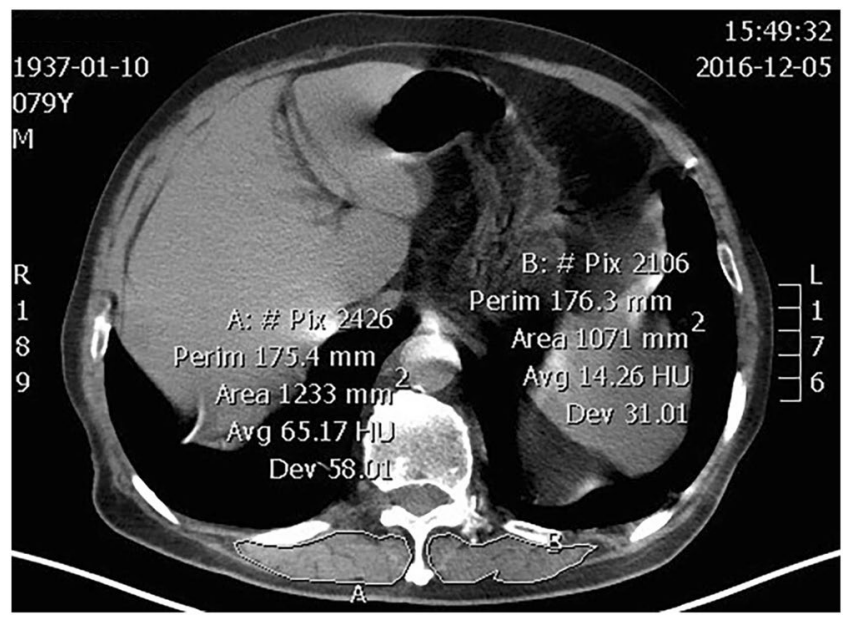

Figure 1. T12DMA of a 79-year-old deceased male patient. The region marked with a solid line indicates the bilateral DMA.

performed using an independent-samples $t$ test. Variables with non-normal distribution were described as median and interquartile range (IQR). The univariate analysis was performed using the Mann-Whitney $U$ test. All qualitative variables were described as number (percentages), and the univariate analysis was performed using the chi-square test.

Pearson coefficients (normally distributed data) or Spearman rho (non-normally distributed data) test were introduced to detect whether T12DMA had any effect on the other laboratory parameters.

Multivariate regression logistic models were constructed to identify the prognostic markers of hospital mortality. The probability of variables entering regression based on the univariate analysis was less than 0.100 .

The selected variables were subjected to forward conditional stepwise regression.

To emphasize the prognostic role of T12DMA in hospital mortality, the receiver operating characteristic (ROC) curve was used to analyze the accuracy, sensitivity, specificity, and area under the ROC. The Youden index was obtained by the ROC analysis, and the maximum tangent point was selected to establish the cutoff value. The Kaplan-Meier curves were drawn to illustrate the effect of T12DMA on the long-term survival between the groups based on the T12DMA cutoff value. The significance analysis was conducted using the log-rank test. Two-tailed $P$ less than 0.05 was considered to be statistically significant.

\section{Results}

Demographic and clinical characteristics. A total of 136 patients were enrolled in this study, including 98 male (72.06\%) and 38 female (27.94\%). The average age was78.00 years (IQR 71.25-83.75). Of the 136 patients, 59 (43.38\%) died at discharge. The demographic and clinical characteristics and details of patient samples in the two groups are shown in Table 1. The acute inflammation biomarkers, such as WBC count (9.64, IQR: 7.06-13.76, $P=0.026)$, NLR (12.81, IQR: 5.58-21.71, $P<0.001)$, PLR (201.30, IQR: 127.71-448.65, $P=0.006)$, had significant differences between the groups. T12DMA (23.10, IQR: 19.16-27.25, $P=0.005)$ was lower in the deceased group than in the surviving group $(P=0.005)$. Further, 105 patients $(77.20 \%)$ had pneumonia at admission, but with no significant differences between the groups. Also, 123 patients (90.4\%) who received IMV during ICU hospitalization showed a bad outcome compared with patients who received NIMV $(P=0.032)$. Patients who received SCS treatment had higher in-hospital mortality compared with non-SCS patients $(P=0.014)$.

Risk factors and survival. As shown in Table 2, the variables in the multivariate regression logistic models were T12DMA [odds ratio $(\mathrm{OR})=0.901 ; 95 \%$ confidence interval $(\mathrm{CI}), 0.841-0.967, P=0.004$ ], NLR $(\mathrm{OR}=1.051 ; 95 \% \mathrm{CI}, 1.019-1.085, P=0.002)$, MV $(\mathrm{OR}=0.190 ; 95 \% \mathrm{CI}, 0.036-0.997, P=0.050)$, and systemic steroid therapy $(\mathrm{OR}=2.439 ; 95 \% \mathrm{CI}, 1.081-5.505, P=0.032)$. They were independent risk factors for predicting the hospital mortality.

Effects of T12DMA on COPD acute exacerbation and long-term survival. T12DMA was associated with the serum albumin level at admission $(r=0.230, P=0.007)$ and the difference between the albumin level at admission and the minimum level of serum albumin during hospitalization $(r=0.240, P=0.005)$ (Table 3 ).

The area under the ROC curve (AUC) for the prognostic role of T12DMA in hospital mortality was 0.666 $(P=0.001)$. The optimal cutoff value of T12DMA to predict survival was $22.515 \mathrm{~cm}^{2}$ (Fig. 2).

The relationship between high/low T12DMA groups based on the cutoff value $\left(22.515 \mathrm{~cm}^{2}\right)$ and overall survival was displayed in the Kaplan-Meier curves (Fig. 3). The median survival was significantly higher in the high-T12DMA group (214 days) than in the low-T12DMA group (32 days) $(P=0.011)$ (Table 4$)$. 


\begin{tabular}{|c|c|c|c|c|c|}
\hline Variable & All patients $(n=136)$ & Surviving $(n=77)$ & Deceased $(n=59)$ & Statistic & $P$ value \\
\hline Age (year) & $78.00(71.25-83.75)$ & $77.00(67.00-83.00)$ & $78.00(73.00-84.00)$ & -1.316 & 0.188 \\
\hline Sex $(\%)$ & & & & 0.341 & 0.559 \\
\hline Male & $98(72.06 \%)$ & $57(74.00 \%)$ & $41(69.50 \%)$ & & \\
\hline Female & $38(27.94 \%)$ & $20(26.00 \%)$ & $18(30.50 \%)$ & & \\
\hline Smoking status (\%) & & & & 2.874 & 0.238 \\
\hline Current smoking & $25(18.40 \%)$ & $11(14.30 \%)$ & $14(23.70 \%)$ & & \\
\hline Quit smoking & $20(14.70 \%)$ & $10(13.00 \%)$ & $10(16.90 \%)$ & & \\
\hline Never smoking & $91(66.90 \%)$ & $56(72.70 \%)$ & $35(59.30 \%)$ & & \\
\hline CCI scores & $2.00(2.00-3.00)$ & $2.00(2.00-3.00)$ & $2.00(2.00-3.00)$ & -0.094 & 0.925 \\
\hline GCS & $11.00(6.00-13.00)$ & $11.00(7.00-14.50)$ & $8.00(6.00-13.00)$ & -1.377 & 0.168 \\
\hline APACHE II & $22.00(20.00-25.00)$ & $22.00(19.50-26.00)$ & $22.00(20.00-25.00)$ & -0.117 & 0.907 \\
\hline $\mathrm{pH}$ & $7.27(7.18-7.36)$ & $7.27(7.17-7.35)$ & $7.28(7.18-7.41)$ & -0.734 & 0.463 \\
\hline $\mathrm{PO}_{2}(\mathrm{~mm} \mathrm{Hg})$ & $75.00(56.00-100.00)$ & $76.00(58.00-111.50)$ & $73.00(54.00-99.00)$ & -1.407 & 0.159 \\
\hline $\mathrm{PCO}_{2}(\mathrm{~mm} \mathrm{Hg})$ & $78.00(54.25-106.25)$ & $77.00(53.50-102.50)$ & $82.00(55.00-113.00)$ & -0.646 & 0.518 \\
\hline $\mathrm{PaO}_{2} / \mathrm{FiO}_{2}(\mathrm{~mm} \mathrm{Hg})$ & $197.06(137.63-266.25)$ & $209.09(145.21-276.89)$ & $193.75(114.00-250.00)$ & -1.164 & 0.245 \\
\hline WBC $\left(\times 10^{9} / \mathrm{L}\right)$ & $9.64(7.06-13.76)$ & $8.67(6.89-11.33)$ & $11.06(8.10-15.30)$ & -2.224 & 0.026 \\
\hline NLR & $12.81(5.58-21.71)$ & $8.80(4.46,18.69)$ & $17.36(8.68,28.00)$ & -3.546 & $<0.001$ \\
\hline PLR & $201.30(127.71-448.65)$ & $180.95(111.45-346.79)$ & $302.13(150.77-561.10)$ & -2.758 & 0.006 \\
\hline $\mathrm{CRP}(\mathrm{mg} / \mathrm{L})$ & $19.97(12.64-50.96)$ & $16.28(11.22-50.90)$ & $30.91(13.05-52.58)$ & -1.383 & 0.167 \\
\hline $\begin{array}{l}\text { Albumin } \\
(\mathrm{g} / \mathrm{L})\end{array}$ & $35.25 \pm 4.98$ & $35.44 \pm 5.11$ & $35.01 \pm 4.84$ & 0.498 & 0.619 \\
\hline $\begin{array}{l}\text { Albumin } \\
(\mathrm{g} / \mathrm{L})\end{array}$ & $28.48 \pm 4.14$ & $28.01 \pm 3.52$ & $29.09 \pm 4.80$ & 1.512 & 0.133 \\
\hline$\Delta$ Albumin $(\mathrm{g} / \mathrm{L})$ & $6.77(3.2-9.25)$ & $6.90(4.15-9.75)$ & $6.0(2.1-9.0)$ & -1.623 & 0.105 \\
\hline T12 DMA $\left(\mathrm{cm}^{2}\right)$ & $23.10(19.16-27.25)$ & $23.82(20.38-28.10)$ & $20.59(16.84-24.95)$ & -2.799 & 0.005 \\
\hline T12 DMD (IU) & $26.52(17.79-33.37)$ & $27.05(18.45-34.80)$ & $24.90(14.88-30.65)$ & -1.693 & 0.091 \\
\hline Pneumonia (\%) & & & & 0.052 & 0.820 \\
\hline No & $31(22.80 \%)$ & $17(22.10 \%)$ & $14(23.70 \%)$ & & \\
\hline Yes & $105(77.20 \%)$ & $60(77.90 \%)$ & $45(76.30 \%)$ & & \\
\hline MV (\%) & & & & 4.587 & 0.032 \\
\hline IMV & $123(90.40 \%)$ & $66(85.70 \%)$ & $57(96.60 \%)$ & & \\
\hline NIMV & $13(9.60 \%)$ & $11(14.30 \%)$ & $2(3.40 \%)$ & & \\
\hline SCS (\%) & & & & 6.000 & 0.014 \\
\hline No & $60(44.10 \%)$ & $41(53.20 \%)$ & $19(32.20 \%)$ & & \\
\hline Yes & $76(55.90 \%)$ & $36(46.80 \%)$ & $40(67.80 \%)$ & & \\
\hline
\end{tabular}

Table 1. Demographic and medical data of the two groups. APACHE: Acute physiology and chronic health evaluation. CCI: Charlson Comorbidity Index. GCS: Glasgow Coma Score. MV: Mechanical ventilation. IMV: Invasive mechanical ventilation. NIMV: Noninvasive mechanical ventilation. NLR: Neutrophil-lymphocyte ratio. PLR: Platelet-lymphocyte ratio. SCS: Systemic corticosteroid. T12DMA: Dorsal muscle group area at the T12 vertebral level. T12DMD: Dorsal muscle group density at the T12 vertebral level.

\section{Discussion}

In this study, aging patients with the advanced stage of COPD, who were admitted to the ICU for MV therapy, had a high hospital mortality rate (43.38\%). The rates of hospital mortality in patients with COPD vary worldwide, ranging from $11 \%$ to $48 \%{ }^{23-25}$. T12DMA, inflammation biomarker NLR, IMV, and administered intravenous steroids were independent risk factors for in-hospital mortality among patients with COPD who were admitted to the ICU. As a surrogate of L3 SMA, T12DMA was used to measure the skeletal muscle mass of patients with COPD for diagnosing sarcopenia. This study showed that low T12DMA affected the in-hospital mortality and long-term survival of patients after ICU admission.

Sarcopenia has a high morbidity in patients with stable COPD, indicating a poor prognosis. This problem should not be neglected in patients referred for physical rehabilitation ${ }^{26,27}$. In addition, COPD exacerbation decreased the endurance of skeletal muscles ${ }^{28}$. The present study focused on critical patients with COPD who were admitted to the ICU. In this stage, the low skeletal muscle mass prognosticated high mortality in the acute episode and chronic rehabilitation stage. COPD-related sarcopenia in the stable stage of the disease was associated with age, GOLD stage ${ }^{7}$, and systemic inflammation ${ }^{27}$. However, in this study, T12DMA had no association with the inflammation biomarkers, including CRP, WBC, PLR, and NLR. Low serum albumin concentrations were associated with reduced muscle mass in relatively healthy, well-nourished, elderly men and women ${ }^{29}$. In the present study, T12DMA was associated with the serum albumin level at admission and the loss during hospitalization, but had no relationship with the minimum level of serum albumin. Puthucheary reported that the cross-sectional area of rectus femoris decreased significantly within the first week ${ }^{12}$. Hence, it was hypothesized 


\begin{tabular}{|c|c|c|c|c|c|c|c|c|}
\hline \multirow{3}{*}{ Step $1^{\mathrm{a}}$} & \multirow[b]{2}{*}{ NLR } & \multirow{2}{*}{$\begin{array}{l}\boldsymbol{B} \\
0.051\end{array}$} & \multirow{2}{*}{\begin{tabular}{|l|} 
SE \\
0.015
\end{tabular}} & \multirow{2}{*}{$\begin{array}{l}\text { Wald } \\
11.667\end{array}$} & \multirow{2}{*}{\begin{tabular}{|l|}
$P$ \\
0.001
\end{tabular}} & \multirow{2}{*}{\begin{tabular}{|l|} 
OR \\
1.053 \\
\end{tabular}} & \multicolumn{2}{|c|}{$95 \% \mathrm{CI}$} \\
\hline & & & & & & & 1.022 & 1.084 \\
\hline & \begin{tabular}{|l|} 
Constant \\
\end{tabular} & -1.108 & 0.295 & 14.074 & 0.000 & 0.330 & & \\
\hline \multirow{3}{*}{ Step $2^{\mathrm{b}}$} & T12DMA & -0.086 & 0.034 & 6.634 & 0.010 & 0.917 & 0.859 & 0.980 \\
\hline & NLR & 0.050 & 0.015 & 10.995 & 0.001 & 1.051 & 1.021 & 1.083 \\
\hline & \begin{tabular}{|l|} 
Constant \\
\end{tabular} & 0.890 & 0.802 & 1.231 & 0.267 & 2.435 & & \\
\hline \multirow{4}{*}{ Step $3^{c}$} & T12DMA & -0.105 & 0.036 & 8.658 & 0.003 & 0.901 & 0.840 & 0.966 \\
\hline & NLR & 0.046 & 0.015 & 9.260 & 0.002 & 1.047 & 1.016 & 1.078 \\
\hline & SCS or not & 1.005 & 0.409 & 6.031 & 0.014 & 2.731 & 1.225 & 6.090 \\
\hline & Constant & 0.790 & 0.815 & 0.942 & 0.332 & 2.204 & & \\
\hline \multirow{5}{*}{ Step $4^{\mathrm{d}}$} & T12DMA & -0.104 & 0.036 & 8.485 & 0.004 & 0.901 & 0.841 & 0.967 \\
\hline & NLR & 0.050 & 0.016 & 9.754 & 0.002 & 1.051 & 1.019 & 1.085 \\
\hline & IMV or not & -1.658 & 0.845 & 3.853 & 0.050 & 0.190 & 0.036 & 0.997 \\
\hline & SCS or not & 0.892 & 0.415 & 4.610 & 0.032 & 2.439 & 1.081 & 5.505 \\
\hline & Constant & 2.568 & 1.202 & 4.560 & 0.033 & 13.037 & & \\
\hline
\end{tabular}

Table 2. Logistic regression analysis of the risk factors for in-hospital mortality. ${ }^{\text {a }}$ Variable(s) entered on step 1: NLR. ${ }^{b}$ Variable(s) entered on step 2: T12DMA. ${ }^{c}$ Variable(s) entered on step 3: SCS or not. ${ }^{\mathrm{d}}$ Variable(s) entered on step 4: IMV or not. Method: forward conditional stepwise regression; the probability of variable entering regression was 0.05 , and the probability of deletion was 0.10 . NLR: Neutrophil-lymphocyte ratio. T12DMA: Dorsal muscle group area at the T12 vertebral level. SCS: Systemic corticosteroid. IMV: Invasive mechanical ventilation.

\begin{tabular}{|c|c|c|}
\hline & $\mathbf{r}$ & $\mathbf{P}$ \\
\hline $\mathrm{pH}$ & -0.055 & 0.522 \\
\hline $\mathrm{PO}_{2}(\mathrm{~mm} \mathrm{Hg})$ & 0.166 & 0.054 \\
\hline $\mathrm{PCO}_{2}(\mathrm{~mm} \mathrm{Hg})$ & 0.02 & 0.819 \\
\hline $\mathrm{PaO}_{2} / \mathrm{FiO}_{2}(\mathrm{~mm} \mathrm{Hg})$ & 0.134 & 0.119 \\
\hline $\mathrm{WBC}\left(\times 10^{9} / \mathrm{L}\right)$ & 0.011 & 0.901 \\
\hline NLR & -0.118 & 0.17 \\
\hline PLR & -0.117 & 0.174 \\
\hline CRP (mg/L) & -0.007 & 0.936 \\
\hline Albumin $_{\mathrm{ad}}(\mathrm{mg} / \mathrm{L})$ & $0.230^{* *}$ & 0.007 \\
\hline Albumin $_{\min }(\mathrm{mg} / \mathrm{L})$ & 0.106 & 0.218 \\
\hline$\Delta$ Albumin $(\mathrm{mg} / \mathrm{L})$ & $0.240^{* * *}$ & 0.005 \\
\hline
\end{tabular}

Table 3. Correlation analysis between T12DMA and laboratory parameters. NLR: Neutrophil-lymphocyte ratio. PLR: Platelet-lymphocyte ratio. CRP: C-reactive protein. Albumin ${ }_{\mathrm{ad}}$ : The serum albumin at admission, Albumin $_{\min }$ : The minimum value during hospitalization, $\Delta$ Albumin: Difference between Albumin ${ }_{\mathrm{ad}}$ and Albumin $_{\text {min }}()$.

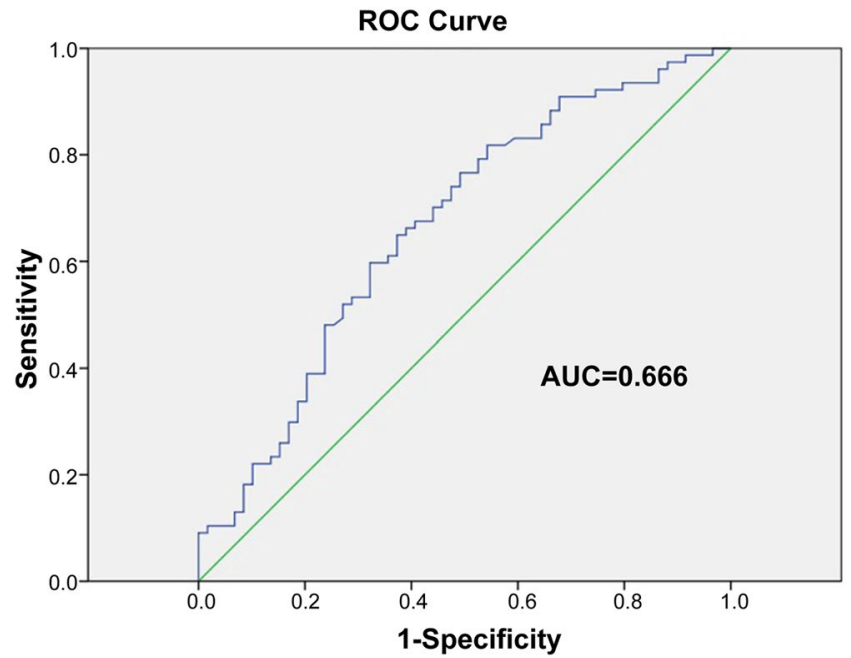

Figure 2. ROC curve of T12DMA to predict the hospital mortality of patients ( $\mathrm{AUC}=0.666, \mathrm{P}=0.005)$. 


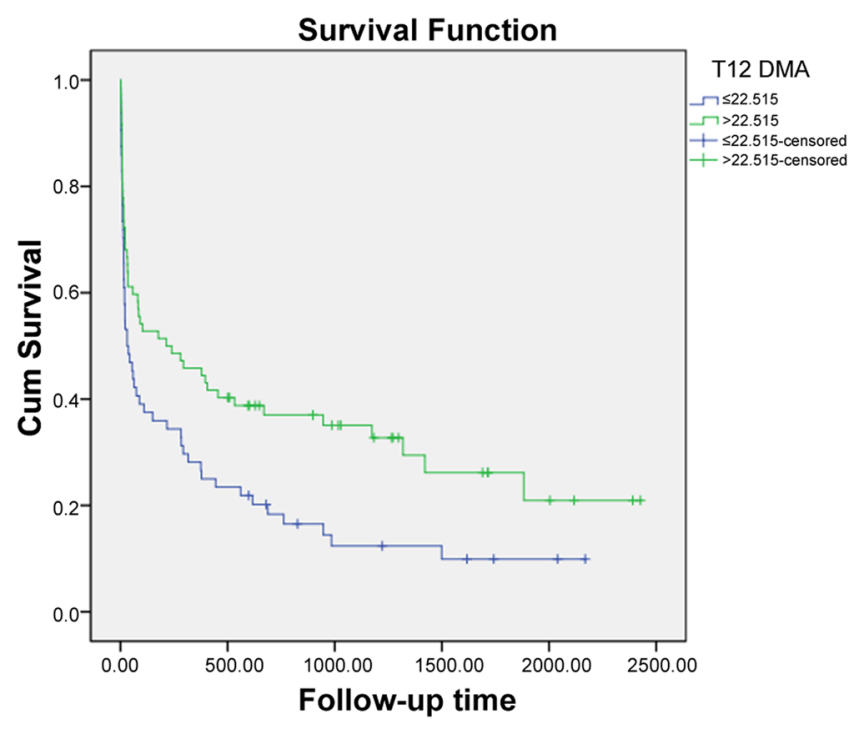

Figure 3. Kaplan-Meier curves of T12DMA to predict long-term survival. The median survival of the highand low-T12DMAs group was 214 days and 32 days, respectively $(\mathrm{P}=0.011)$.

\begin{tabular}{|l|l|l|l|}
\hline & $\begin{array}{l}\text { Median survival } \\
(\mathbf{d})\end{array}$ & $\chi^{2}$ & $\boldsymbol{P}$ \\
\hline$\leq 22.515\left(\mathrm{~cm}^{2}\right)$ & 31 & 6.454 & 0.011 \\
\hline$>22.515\left(\mathrm{~cm}^{2}\right)$ & 214 & & \\
\hline
\end{tabular}

Table 4. Log-rank analysis between T12DMA and long-term survival.

that critical patients with COPD had skeletal muscle mass loss due to ICU admission and MV therapy. Despite no significant difference between the two groups, the serum albumin level significantly declined in all patients. Muscle mass is maintained by protein homeostasis ${ }^{30}$, with serum albumin being the most relevant. A high skeletal muscle mass at admission may maintain the protein homeostasis for critically ill patients. This hypothesis should be verified in prospective studies. Puthucheary et al. ${ }^{31}$ investigated the metabolic phenotype of skeletal muscle wasting in early critical illness, suggesting that non-fat sources and removal of fatty acid supplementation could improve the skeletal muscle energy status.

Further, $50.6 \%$ patients had COPD complicated with pneumonia during hospitalization ${ }^{32}$. In the present study, $77.2 \%$ patients with COPD, who were admitted to the ICU, had co-existing pneumonia. The majority of AECOPD events were attributed to bacterial or viral infection. This study showed that WBC count, NLR, and PLR, as inflammation biomarkers, were significantly elevated in the deceased group. Especially NLR within $24 \mathrm{~h}$ after admission to the ICU was an independent risk factor for predicting the in-hospital mortality. In line with Terradas et al. ${ }^{33}$, NLR $>7$ was a predictive factor for AECOPD in-hospital mortality. The elevated NLR affected readmission and mortality in the 6-month follow-up period ${ }^{34}$. As demonstrated by Valiollah Amri Malehm, the mortality rate in IMV (54\%) was higher than that in NIMV (8\%) in an observational cohort study. The mortality rate in IMV and NIMV was $46.34 \%$ and $15.38 \%$, respectively. Furthermore, IMV was an independent predictive factor for in-hospital mortality in our study.

Systemic steroid therapy is commonly used for hospitalized patients with AECOPD. This study showed that the intravenous administration of steroids was an independent risk factor for hospital mortality. Bahloul et al. ${ }^{35}$ confirmed that systemic corticosteroid therapy could reduce the duration of MV and shorten the ICU LOS. McCann et al. ${ }^{36}$ indicated that the prescribed maintenance steroid dose was not associated with the duration of MV or ICU LOS. A meta-analysis performed by Abroug et al. ${ }^{37}$ showed no beneficial effect of corticosteroids in critically ill patients. Another open-label study conducted in two ICUs in Tunisia also found negative outcomes ${ }^{38}$. This study provided a negative evidence for aging patients with COPD requiring MV.

This study had several limitations. This was a single-center retrospective study. A certain number of patients were eliminated to analyze the skeletal muscle mass on CT images. Especially very critically ill patients who could not undergo CT often had a bad outcome rapidly within $24 \mathrm{~h}$; they should be excluded from the study according to the study design. Deleting the information on these patients had a minimal effect on the study results. Only 136 patients were enrolled in the study. Insufficient sample size, especially the fewer number of female patients, might have introduced some bias in the results. Hence, large-scale studies with more patients from other centers should be conducted in the future. Besides, this was a retrospective study. Hence, the CT scans after ICU admission were not available for all patients to measure the skeletal muscle mass loss during ICU hospitalization. It was hypothesized that critical patients with COPD had skeletal muscle mass loss during ICU hospitalization according to the sharp decline in serum albumin levels. The acute loss of skeletal muscle 
mass loss and target nutrition supply among patients with COPD during the episodes of acute exacerbation and ICU admission for MV therapy should be further investigated.

\section{Conclusions}

T12DMA was an independent predictive factor for hospital mortality and a predictor of long-term survival in elderly patients with COPD who were admitted to the ICU. Skeletal muscle mass loss was a crucial problem in the stable and advanced stages of COPD and hence should not be neglected. This study presented an alternative method for measuring the skeletal muscle mass when the CT scans were available, especially for critically ill patients whose body composition like BMI could not be acquired. The clinical parameters, such as NLR, IMV, and systemic steroid therapy, were independent risk factors for predicting the hospital mortality. Large-sample, multi-center studies, involving more female patients, are required in the future to clarify the reasons behind sex-specific difference in findings.

\section{Data availability}

All data generated or analysed during this study are included in this published article (and its Supplementary Information files)

Received: 22 August 2019; Accepted: 2 December 2019;

Published online: 13 December 2019

\section{References}

1. Collaborators, G. B. D. C. R. D. Global, regional, and national deaths, prevalence, disability-adjusted life years, and years lived with disability for chronic obstructive pulmonary disease and asthma, 1990-2015: a systematic analysis for the Global Burden of Disease Study 2015. Lancet. Respir Med. 5, 691-706 (2017).

2. Zhou, M. et al. Mortality, morbidity, and risk factors in China and its provinces, 1990-2017: a systematic analysis for the Global Burden of Disease Study 2017. Lancet. (2019).

3. Hanania, N. A., Sharma, G. \& Sharafkhaneh, A. COPD in the elderly patient. Semin Respir Crit Care Med. 31, 596-606 (2010).

4. Rabe, K. F. \& Watz, H. Chronic obstructive pulmonary disease. Lancet. 389, 1931-1940 (2017).

5. Munhoz da Rocha Lemos Costa, T. et al. Body composition and sarcopenia in patients with chronic obstructive pulmonary disease. Endocrine. 60, 95-102 (2018).

6. Costa, T. M. et al. Sarcopenia in COPD: relationship with COPD severity and prognosis. J Bras Pneumol. 41, 415-421 (2015).

7. Limpawattana, P. et al. Sarcopenia in chronic obstructive pulmonary disease: A study of prevalence and associated factors in the Southeast Asian population. Chronic respiratory disease. 15, 250-257 (2018).

8. Sheean, P. M. et al. The prevalence of sarcopenia in patients with respiratory failure classified as normally nourished using computed tomography and subjective global assessment. JPEN J Parenter Enteral Nutr. 38, 873-879 (2014).

9. Moisey, L. L. et al. Skeletal muscle predicts ventilator-free days, ICU-free days, and mortality in elderly ICU patients. Crit Care. 17, R206 (2013).

10. Weijs, P. J. et al. Low skeletal muscle area is a risk factor for mortality in mechanically ventilated critically ill patients. Crit Care. $\mathbf{1 8}$, R12 (2014).

11. Looijaard, W. G. et al. Skeletal muscle quality as assessed by CT-derived skeletal muscle density is associated with 6-month mortality in mechanically ventilated critically ill patients. Crit Care. 20, 386 (2016).

12. Puthucheary, Z. A. et al. Acute skeletal muscle wasting in critical illness. Jama. 310, 1591-1600 (2013).

13. Herridge, M. S. et al. Functional disability 5 years after acute respiratory distress syndrome. The New England journal of medicine. 364, 1293-1304 (2011).

14. Batt, J., dos Santos, C. C., Cameron, J. I. \& Herridge, M. S. Intensive care unit-acquired weakness: clinical phenotypes and molecular mechanisms. Am J Respir Crit Care Med. 187, 238-246 (2013).

15. Pfoh, E. R. et al. Physical declines occurring after hospital discharge in ARDS survivors: a 5-year longitudinal study. Intensive care medicine. 42, 1557-1566 (2016).

16. Cruz-Jentoft, A. J. et al. Sarcopenia: revised European consensus on definition and diagnosis. Age Ageing. 48, 16-31 (2019).

17. Derstine, B. A. et al. Skeletal muscle cutoff values for sarcopenia diagnosis using T10 to L5 measurements in a healthy US population. Sci Rep. 8, 11369 (2018).

18. Boutin, R. D. et al. CT of Patients With Hip Fracture: Muscle Size and Attenuation Help Predict Mortality. AJR Am J Roentgenol. 208, W208-W215 (2017).

19. Lee, C. S. et al. Dorsal muscle group area and surgical outcomes in liver transplantation. Clin Transplant. 28, 1092-1098 (2014).

20. Canvasser, L. D. et al. Paraspinous muscle as a predictor of surgical outcome. J Surg Res. 192, 76-81 (2014).

21. Charlson, M. E., Pompei, P., Ales, K. L. \& MacKenzie, C. R. A new method of classifying prognostic comorbidity in longitudinal studies: development and validation. J Chronic Dis. 40, 373-383 (1987).

22. Mowery, N. T. Ventilator Strategies for Chronic Obstructive Pulmonary Disease and Acute Respiratory Distress Syndrome. The Surgical clinics of North America. 97, 1381-1397 (2017).

23. Alaithan, A. M., Memon, J. I., Rehmani, R. S., Qureshi, A. A. \& Salam, A. Chronic obstructive pulmonary disease: hospital and intensive care unit outcomes in the Kingdom of Saudi Arabia. Int J Chron Obstruct Pulmon Dis. 7, 819-823 (2012).

24. Feng, Z. et al. Efficacy of Various Scoring Systems for Predicting the 28-Day Survival Rate among Patients with Acute Exacerbation of Chronic Obstructive Pulmonary Disease Requiring Emergency Intensive Care. Can Respir J. 2017, 3063510 (2017).

25. He, H., Sun, Y., Sun, B. \& Zhan, Q. Application of a parametric model in the mortality risk analysis of ICU patients with severe COPD. Clin Respir J. 12, 491-498 (2018).

26. Davalos-Yerovi, V. et al. Sarcopenia According to the Revised European Consensus on Definition and Diagnosis (EWGSOP2) Criteria Predicts Hospitalizations and Long-Term Mortality in Rehabilitation Patients With Stable Chronic Obstructive Pulmonary Disease. Journal of the American Medical Directors Association. 20, 1047-1049 (2019).

27. Byun, M. K., Cho, E. N., Chang, J., Ahn, C. M. \& Kim, H. J. Sarcopenia correlates with systemic inflammation in COPD. Int J Chron Obstruct Pulmon Dis. 12, 669-675 (2017).

28. Poberezhets, V., Mostovoy, Y. \& Demchuk, H. Exacerbation of chronic obstructive pulmonary diseases as a risk factor of the skeletal muscle dysfunction. Lung India: official organ of Indian Chest Society. 36, 188-192 (2019).

29. Baumgartner, R. N., Koehler, K. M., Romero, L. \& Garry, P. J. Serum albumin is associated with skeletal muscle in elderly men and women. The American journal of clinical nutrition. 64, 552-558 (1996).

30. Bear, D. E., Parry, S. M. \& Puthucheary, Z. A. Can the critically ill patient generate sufficient energy to facilitate exercise in the ICU? Current opinion in clinical nutrition and metabolic care. 21, 110-115 (2018). 
31. Puthucheary, Z. A. et al. Metabolic phenotype of skeletal muscle in early critical illness. Thorax. 73, 926-935 (2018).

32. Yu, S., Fang, Q. \& Li, Y. Independent factors associated with pneumonia among hospitalized patients with acute exacerbations of chronic obstructive pulmonary disease. Medicine. 97, e12844 (2018).

33. Terradas, R. et al. Eosinophil count and neutrophil-lymphocyte count ratio as prognostic markers in patients with bacteremia: a retrospective cohort study. PloS one. 7, e42860 (2012).

34. Duman, D. et al. The utility of inflammatory markers to predict readmissions and mortality in COPD cases with or without eosinophilia. Int J Chron Obstruct Pulmon Dis. 10, 2469-2478 (2015).

35. Bahloul, M. et al. Efficacy of corticosteroid therapy in severe decompensation of chronic obstructive pulmonary disease requiring mechanical ventilation. Am J Ther. 20, 630-637 (2013).

36. McCann, J., Teare, K., Cochard, E. \& Toney, B. Relationship of Steroid Dosing and Duration of Mechanical Ventilation in Adult Patients With Acute Exacerbations of Chronic Obstructive Pulmonary Disease. J Pharm Pract. 31, 157-162 (2018).

37. Abroug, F. et al. Systemic corticosteroids in acute exacerbation of COPD: a meta-analysis of controlled studies with emphasis on ICU patients. Ann Intensive Care. 4, 32 (2014).

38. Abroug, F. et al. Prednisone in COPD exacerbation requiring ventilatory support: an open-label randomised evaluation. Eur Respir J. 43, 717-724 (2014).

\section{Acknowledgements}

The authors thank the Department of Radiology for the support in reviewing the CT scans. This study was funded by the 2016 Yangzhou Key Research and Development Plan - Social Development (YZ2016062).

\section{Author contributions}

Jiehua Zhi: acquisition of data, analysis and interpretation of data; revising it critically for important intellectual content; Given final approval of the version to be published; Agreed to be accountable for all aspects of the work in ensuring that questions related to the accuracy. Qing Shan: substantial contributions to conception and design; revising it critically for important intellectual content; Given final approval of the version to be published; integrity of any part of the work are appropriately investigated and resolved. Lanyu Liang: substantial contributions to conception and design; analysis and interpretation of data; Been involved in drafting the manuscript; Given final approval of the version to be published; Agreed to be accountable for all aspects of the work in ensuring that questions related to the accuracy. Han Liu: acquisition of data; revising it critically for important intellectual content; Given final approval of the version to be published; integrity of any part of the work are appropriately investigated and resolved. Hua Huang: acquisition of data; Given final approval of the version to be published; integrity of any part of the work are appropriately investigated and resolved.

\section{Competing interests}

The authors declare no competing interests.

\section{Additional information}

Correspondence and requests for materials should be addressed to L.L.

Reprints and permissions information is available at www.nature.com/reprints.

Publisher's note Springer Nature remains neutral with regard to jurisdictional claims in published maps and institutional affiliations.

(c) (1) Open Access This article is licensed under a Creative Commons Attribution 4.0 International License, which permits use, sharing, adaptation, distribution and reproduction in any medium or format, as long as you give appropriate credit to the original author(s) and the source, provide a link to the Creative Commons license, and indicate if changes were made. The images or other third party material in this article are included in the article's Creative Commons license, unless indicated otherwise in a credit line to the material. If material is not included in the article's Creative Commons license and your intended use is not permitted by statutory regulation or exceeds the permitted use, you will need to obtain permission directly from the copyright holder. To view a copy of this license, visit http://creativecommons.org/licenses/by/4.0/.

(C) The Author(s) 2019 